
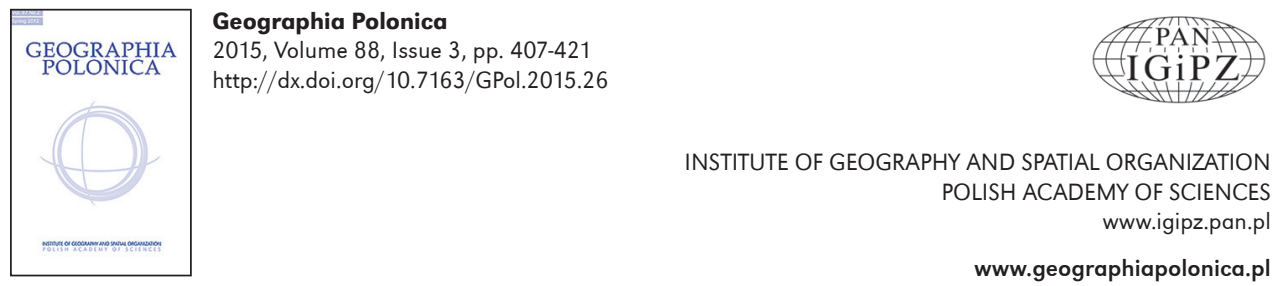

\title{
AUTOMATIC BUILDING EXTRACTION BASED ON MULTIRESOLUTION SEGMENTATION USING REMOTE SENSING DATA
}

\author{
Neeti Shrivastava ${ }^{1}$ - Praveen Kumar Rai ${ }^{2}$ \\ ${ }^{1}$ Indian Institute of Remote Sensing, Dehradun \\ Uttrakhand: India \\ e-mail: neeti.iirs@gmail.com \\ ${ }^{2}$ Department of Geography \\ Banaras Hindu University \\ Varanasi-221005, Uttar Pradesh: India \\ e-mail: rai.vns82@gmail.com
}

\begin{abstract}
Analysis of high resolution remote sensing images, included in the object-oriented approach, involved classifying the image objects according to class descriptions organised in an appropriate knowledge base. This technique is created by means of inheritance mechanisms, concepts, and methods of fuzzy logic and semantic modeling. The process of the object oriented classification mainly involved two sections: multiresolution segmentation and image classification. Multiresolution segmentation is a new procedure for image object extraction. It allows the segmentation of an image into a network of homogeneous image regions at any chosen resolution. These image object primitives represent image information in an abstract form, serving as building blocks and information carries for subsequent classification. A study was taken up to perform object oriented fuzzy classification using high resolution satellite data (Cartosat-1 fused with IRS-1C, LISS IV data) for automatic building extraction in the study area covering the administrative area of BHEL (Bharat Heavy Electrical Limited) colony, Haridwar, Uttrakhand (India). The study area was located at $29^{\circ} 56^{\prime} 55.51^{\prime \prime} \mathrm{N}$ to $29^{\circ} 56^{\prime} 11.49^{\prime \prime} \mathrm{N}$ latitude and $78^{\circ} 05^{\prime} 42.45^{\prime \prime} \mathrm{E}$ to $78^{\circ} 07^{\prime} 00.09^{\prime \prime} \mathrm{E}$ longitude. Two approaches were used: applying different spatial filters, and object orientation. The merged image is filtered using different high pass filters, such as: Kirsch, Laplace, Prewitt, Sobel, and Canny filtered images. The overall accuracy of the classified image was 0.93 , and Kappa accuracy was 0.89 . The produced accuracy for buildings, vegetation, and shadows were $0.9545,1.0$, and 0.8888 , respectively, whereas user accuracy for buildings vegetation, and shadows were $1.0,0.9375$, and 1.0 , respectively. Overall classification accuracy was based on TA mask (training and test area mask) and it was 0.97 . Kappa accuracy was 0.95 .
\end{abstract}

\section{Key words}

building extraction $\cdot$ high resolution data $\cdot$ multiresolution segmentation $\bullet$ object oriented fuzzy classification - spatial filter 


\section{Introduction}

Land use in urban areas changes continuously mainly due to the construction of new buildings, roads, and other man-made objects. Map content should be regularly updated to include the changes. The building region is one of the important land types in land cover classification. The extracted buildings are useful for disaster monitoring and building reconstruction, as well as other applications, such as urban planning, and telecommunication. Urban areas are rapidly changing. The changes are mainly due to human activities in construction, destruction, or extension of topographic elements such as buildings and roads. These changes in the urban environment mean that old records must be kept updated. Planners can then count on having available, accurate building zones for urban planning, maintenance, and development (Pandey 2004). Although automatic building extraction has great importance in city planning and for natural disaster and crisis management, it remains a complex problem for scientists. There are problems encountered in building extraction approaches. The main problem is confusing the building class with other object classes, such as shadows, vegetation, and the ground. The detection of a non-building as a building, and mixing together trees and shadows are examples of other misclassification problems. These misclassification problems, which are attributable to a single dataset and method, have a negative effect on the accuracy of the classification process. For this reason, different approaches and methods have been proposed to solve the problems caused by the complexity of the classification process (Uzar 2014). Object-based image analysis, or geographic object-based image analysis, is an emerging field resulting from new earth observation techniques and concepts, and has received considerable impetus over the last decade (Blaschke 2010). Object-based analysis is formally defined as "a sub-discipline of Geographic Information Science (GIScience) devoted to developing automated methods to partition remote sensing imagery into meaningful image-objects, and assessing their characteristics through spatial, spectral, and temporal scales, so as to generate new geographic information in GIS-ready format" (Hay \& Castilla 2008: 77).

There are many different approaches for building and road extraction from high-resolution remote sensing imagery. At present, template matching and snakes are methods for roads extraction based on the spectrum feature (Hu et al. 2002; Ding et al. 2010). But for high resolution satellite images, the phenomenon where the same things have different spectrums and the same spectrums belong to different things, is very obvious. The traditional methods could not solve the problem. Automatic building and road extraction algorithm can reduce both time and labor to construct and update the road spatial database in such applications. However, fully automated algorithms to recognise the road for applications where accuracy is critical are very difficult (Jeon et al. 2002; Tupin et al. 2002; Chaudhuri et al. 2012; Teng et al. 2014).

Remote sensing is very important when used for collecting geographical data. Huge remote sensing images have been increasing rapidly. Currently, thousands of GB remote sensing images can be generated and need to be managed. High resolution remote sensing images are vital for national defense, disaster relief, and so on (Bruzzone et al. 2006). The automated or semi-automated analysis of these images has been obstructed by the high complexity of such images (Gupta \& Bhadauria 2014). The datasets obtained from different sensor systems create the opportunity for the development of methods to extract objects (Baltsavias 1999; Tarsha-Kurdi et al. 2007; Matikainen et al. 2009; Rottensteiner 2012). Regarding the data source, due to the limitations of using single-source data, the integration of multi-sensor data is desired because this method preserves the many advantages of the involved datasets (Gruen 2008; Kwak et al. 2012).

High resolution satellite images provide a valuable new data source for geographic 
information acquisition. Building detection from high resolution satellite images has attracted great attention in recent years. To automate the process and produce reliable, precise, and complete datasets, multiple data sources and advanced techniques should be used (Lee et al. 2003). The increasing availability of the high spatial resolution satellite images has provided a new data source for building extraction. When compared with the aerial photographs, the high resolution satellite images provide several advantages that include cost and accessibility. A number of recent studies have used the spectral reflectance values to detect buildings (Shan \& Lee 2002).

Geographic data acquisition is usually very time consuming and costly (Chang 2007). Thus, many Geographic Information System (GIS) applications suffer from the lack of current land cover use information. This problem could be overcome by using remote sensing technology. Data acquisition by remote sensing for mapping and GIS has traditionally been characterised by efficient and accurate manual extraction. But, manual information extraction is time consuming and requires qualified people. Speeding up this process with the use of automatic or semiautomatic feature extraction techniques has become a necessity. Using a combination of multiple data sources and the integration of images with the other data sources such as DSMs, existing GIS data, ground plans, and prior knowledge, appears to be a new trend in building extraction.

Automatic extraction of urban buildings from high resolution satellite images has been an active research subject for a decade. Most early works used black and white aerial images as a single data source (Huertas \& Nevatia 1988; Lin \& Nevatia 1998). These methods are mainly based on edge detection, line extraction, and building polygon generation. Several approaches have used the building models to facilitate and automate the building extraction procedure (Tseng \& Wang 2003). Research in feature extraction is still very diverse and object extraction is a fundamental computer vision operator. There are different methodologies for feature extraction, especially for linear features such as image fusion for feature extraction (Pigeon et al. 2001), the fuzzy-based approach (Agouris et al. 1998), mathematical morphology (Zhang et al. 1999), the model based approach (Bückner 1998), dynamic programming, multi-scale grouping and context (Mayer et al. 1997), and kalman filtering.

The strong motivation to develop techniques for the extraction of image objects stems from the fact that most image data exhibit a characteristic texture which is neglected in common classifications. This is true despite the fact that scientists had already started to tackle this problem in the 1970s (Kettig \& Landgrebe 1976; Burnett \& Blaschke 2002). Human visual perception involves a set of processes for distinguishing top-down attention from the stimulus-driven bottom up (Itti \& Koch 2001).

Most of the recent work on building extraction from high resolution satellite images is based on supervised techniques. These techniques either require a digital image processing classification method based on initial training data to provide hypotheses for the positions and sizes of the candidate building features (Lin \& Nevatia 1998; Benediktsson et al. 2003), or they use training sets or model databases to classify or match the buildings (Kim \& Nevatia 1999; Segl \& Kaufmann 2001). Supervised classification is one of the most commonly undertaken analyses of remotely sensed data. The output of a supervised classification is effectively a thematic map that provides a snapshot representation of the spatial distribution of a particular theme of interest such as land cover. The goal of a supervised image classification system is to group images into semantic categories, giving the opportunity for fast and accurate image search (Rizvi \& Mohan 2010).

The main steps in digital image processing are: (a) preprocessing, which is a data preparation step for contrast enhancement, noise reduction or filtering (Chen 1998; Gonzalez \& Woods 2002), (b) feature extraction, 
for retrieving non-redundant and significant information from an image. This operation is targeted at achieving time efficiency at the cost of data reduction (Pal \& Pal 1993; Lucchese \& Mitra 2001) followed by object detection, localisation, and recognition, which determine the position, location, and orientation of objects (Foresti \& Pellegrino 2004).

The new availability of very high resolution satellite images offers a mapping potential for scales reaching from $1: 5,000$ to $1: 10,000$ (Puissant \& Weber 2002). Classification of high-resolution satellite images using standard per-pixel approaches is difficult because of the high volume of data, as well as the high spatial variability within the objects. Segmentation of the images is carried out using the region based algorithms, such as morphological marker based watershed transformation, by employing the advantages of multiresolution framework and multiscale gradient algorithms. The segmentation of the color images is obtained using watershed transformation to get homogenous regions. The classification technique is then applied to these homogenous regions taking the shape, texture, and spectral properties of the regions (Rizvi \& Mohan 2010).

Man-made objects such as buildings can be easily detected. The research in this domain expect significant results in the field of remote sensing in urban areas. However, the real potentialities dealing with such high resolution image data remain relatively unknown. Problems and difficulties appear when extracting objects with a high, local-variance context and spectral signatures disturbances (Lhomme et al. 2004). Thus, the extraction methods should be adapted to these new images. Problems and difficulties appear when extracting objects which have a high, local-variance context and spectral signature disturbances (Lhomme et al. 2004). Thus, the extraction methods should be adapted to these new images.

The main objectives of this paper are to analyse the result of automatic extraction of buildings from high resolution imagery, and processing of images using edge detectors.

\section{Study area}

The study area covered the administrative area of BHEL (Bharat Heavy Electrical Limited) colony, Haridwar, Uttrakhand. In India, $\mathrm{BHEL}$ is the largest engineering and manufacturing enterprise in the energy-related infrastructure sector, today. Bharat Heavy Electrical Limited was established more than 40 years ago, and was founded in the 1950s. Its operations are organised around three business sectors: Power, Industry including Transmission, Transportation, and Telecommunication and Renewable energy. The company has been earning profits continuously since 1971-1972 and paying dividends since 1976-1977. The geographical location of the study is shown in the Figure 1.

\section{Software and data used}

In this paper the following software was used: Erdas Imagine (ver. 10), Definiens Developer (eCognition ver. 8), ArcGIS (ver. 9.3), and MS Office.

The Cartosat-1 spacecraft is configured with Panchromatic cameras which are mounted so that one camera is looking at +26 degree with respect to nadir and the other at -5 degree with respect to nadir along the track. These two cameras combined, provide stereoscopic image pairs in the same pass. Also, the whole spacecraft is steerable across track to provide wider coverage in a shorter period. A brief description of the payload and the other mainframe elements are given in Tables 1 and 2. Satellite data used in this study is georeferenced with UTM-zone 44 projection system and WGS-84 datum.

\section{Methodology}

The process of automatic feature extraction is mainly divided into three parts: (a) image processing, (b) information extraction using spatial filtering, (c) information extraction using fuzzy rule based classification. 


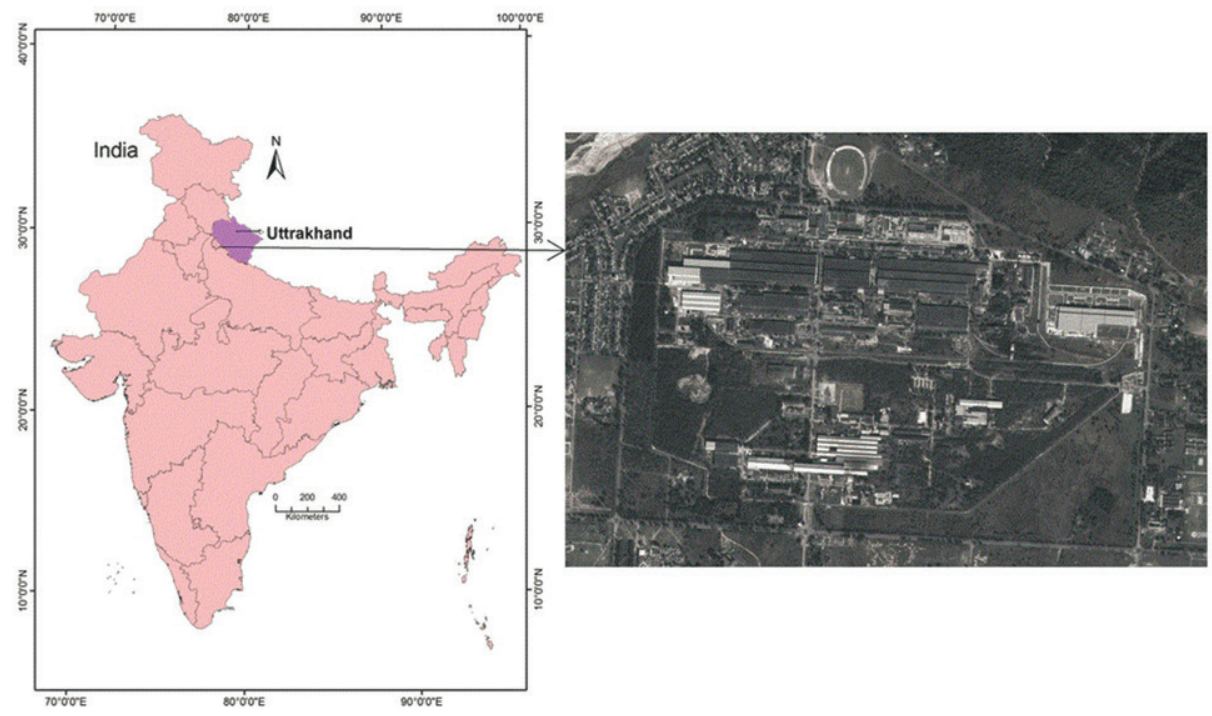

Figure 1. Geographical location of the study area in the Haridwar district, Uttrakhand (India) shown in satellite data

Table 1. Orbit specifications of Cartosat-1

\begin{tabular}{|c|l|l|}
\hline No. & \multicolumn{1}{|c|}{ Orbit Characteristic } & \multicolumn{1}{|c|}{ Specification } \\
\hline 1 & Nominal altitude & $617.99 \mathrm{~km}$ \\
2 & Number of orbits per day & 15 \\
3 & Orbital repeativity cycle & 116 days \\
4 & Local time for Equatorial & $10: 30 \mathrm{am}$ \\
& crossing & \\
5 & Orbital parameters: & $6996.12 \mathrm{~km}$ \\
& - Semi major axis & 0.001 \\
& - Eccentricity & 97.87 degree \\
\hline
\end{tabular}

The panchromatic image of the study area was fused with the multispectral images using a Brovey transformation and the nearest neighborhood method. There are other methods for the image fusion-like principle component analysis. They are Intensity Hue and Saturation (IHS), and multiplicative. All these methods have been tested for better output but out of these methods, the Brovey Transform was found to be the best for the study purposes. Multiplicative is also used for the urban application. Details of methodology for automatic feature extractions are given in Figure 2.

The fuzzy classification method takes into account that there are pixels of mixed makeup, that is, pixels cannot be definitively assigned to one category. "Clearly, there needs to be a way to make the classification algorithms more sensitive to the imprecise (fuzzy) nature of the real world" (Jensen 1996). Fuzzy classification works using a membership function, where a pixel value is determined by whether it is closer to one class than another. A fuzzy classification does not have definite boundaries, and each pixel can belong to several different classes (Jensen 1996).

Two approaches are used for automatic feature extraction in the study area: (1) applying different spatial filters and (2) object oriented fuzzy classification. The concept of object based information extraction is that to interpret an image, the relevant semantic information is represented by meaningful image objects and their mutual relationship rather than individual pixels (Gupta \& Bhadauria 2014).

\section{Approach 1}

Filtering is a broad term, which refers to the altering of spatial or spectral features for image enhancement. Convolution filtering 
Table 2. Cartosat-1 payload specification

\begin{tabular}{|c|c|c|}
\hline No. & Parameter Name & $\begin{array}{l}\text { Specification Fore }(+26 \mathrm{deg}) \text { and } \\
\text { Aft (-5 deg) }\end{array}$ \\
\hline 1 & $\begin{array}{l}\text { Spatial resolution: } \\
\text { GIFOV (across-track } \times \text { along track) }\end{array}$ & $2.5 \times 2.78 \mathrm{~m}$ (Fore); $2.22 \times 2.23 \mathrm{~m}($ Aft $)$ \\
\hline 2 & $\begin{array}{l}\text { Spectral resolution: } \\
\text { - No. of bands } \\
\text { - Bandwidth }\end{array}$ & $\begin{array}{l}1 \text { Panchromatic } \\
500 \mathrm{~nm} \text { to } 850 \mathrm{~nm}\end{array}$ \\
\hline 3 & $\begin{array}{l}\text { Radiometric resolution: } \\
\text { - Saturation radiance } \\
\text { - Quantisation } \\
\text { - SNR }\end{array}$ & $\begin{array}{l}55 \mathrm{mw} / \mathrm{cm}^{\star} \mathrm{cm} / \mathrm{str} / \text { micron } \\
10 \mathrm{bits} \\
345 \text { at saturation radiance }\end{array}$ \\
\hline 4 & Swath (stereo) Fore + Aft combined (mono) & $30 \mathrm{~km} 26.855 \mathrm{~km}$ \\
\hline 5 & $\begin{array}{l}\text { CCD parameters: } \\
\text { - No. of detectors / elements } \\
\text { - Detector element size } \\
\text { - Odd-Even Spacing }\end{array}$ & $\begin{array}{l}12,000 \text { per camera } \\
7 \times 7 \text { microns } \\
35 \text { microns staggered }\end{array}$ \\
\hline 6 & $\begin{array}{l}\text { Optics: } \\
\text { - No. of mirrors } \\
\text { - Effective focal length (mm) } \\
\text { - F-Number } \\
\text { - Field of view (degrees) }\end{array}$ & $\begin{array}{l}3 \\
1980 \\
F / 4.5 \\
\pm 1.08\end{array}$ \\
\hline 7 & Integration time (ms) & 0.336 \\
\hline 8 & Nominal B/H ratio for stereo & 0.62 \\
\hline
\end{tabular}

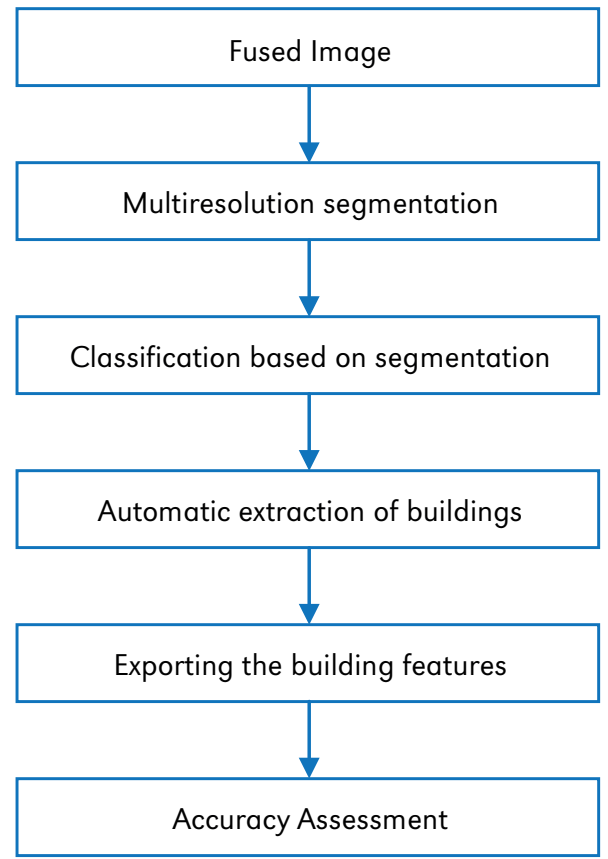

Figure 2. Flow chart of automatic feature extraction 
is the process of averaging small sets of pixels across an image. Convolution filtering is used to change the spatial frequency characteristics of an image (Jensen 1996).

The merged image is filtered using different high pass filters like Kirsch, Laplace, Prewitt, Sobel, and Canny filtered images (Fig. 3 a-e). These filters are used for the detection of the edges but were not found appropriate for the study area. For this reason, this method was discarded and another approach was used which was suitable for detection of the edge.

\section{Approach 2}

Multiresolution segmentation is a new procedure for image object extraction. It allows for the segmentation of an image into a network of homogeneous image regions at any chosen resolution (Pandey 2004; Dell'Acqua \& Gamba 2007). These image object primitives represent image information in an abstract form serving as building blocks and information carries for subsequent classification. Beyond purely spectral information, image objects contain a lot of additional attributes which can be used for classification. Image objects offer some basic advantages:

- Multiresolution segmentation separates adjacent regions in an image as long as they are significantly contrasted, even when the regions themselves are characterised by a certain texture or noise. Thus, even textured data can be analysed.

- Homogeneous image objects provide a significantly increased signal-to-noise ratio compared to single pixels, as to the attributes to be used for classification. Thus, independent of the multitude
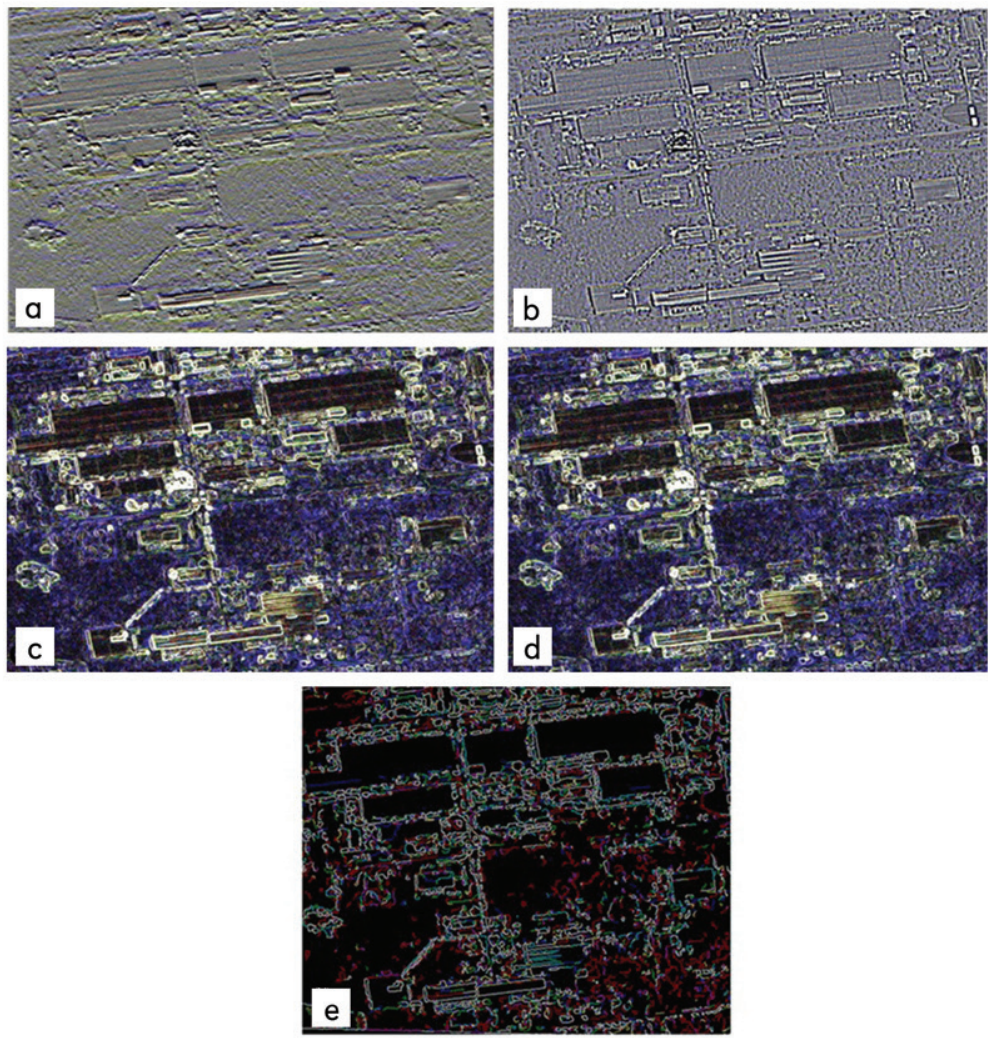

Figure 3 (a-e). Filtered images (Kirsch, Laplace, Prewitt, Sobel, Canny filtered images) 
of additional information, the classification is more robust.

- Each classification task has its specific scale. Only image objects of an appropriate resolution permit analysis of meaningful contextual information. Multiresolution segmentation provides the possibility to easily adapt image object resolution to specific requirements, data and tasks.

- Segmentation drastically reduces the sheer number of units to be handled for classification. Even if a lot of intelligence is applied to the analysis of each single image object, the classification works relativity fast.

- Using the possibility to produce image objects in different resolutions, a project can contain a hierarchical network with different object levels of different resolutions. This structure represents image information on different scales simultaneously. Thus, different object levels can be analysed in relation to each other. For instance, image objects can be classified as to the detailed composition of sub-objects.

- The object oriented approach which first extracts homogeneous regions and then classifies them, avoids the annoying salt and pepper effect of the more or less spatially finely distributed classification results, which are typical of pixel based analysis.

\section{Result and Discussion}

\section{Multiresolution segmentation}

First of all, the Cartosat-1 fused image was segmented using Definiens Developer. The building segmentation was carried out to adjust the scale parameter, shape factor, and compactness. These parameters should be adjusted so that the process gives a homogeneous region with a defined boundary of the object of interest. Once these parameters' ratios are adjusted into a homogeneous pattern, different rules can be implemented using the fuzzy rule base for feature extraction.
Table 3 shows the parameters used for segmentation. The process of segmentation by scale parameters 17,30 , and 40 were used, respectively.

Table 3. Parameters used for segmentation

\begin{tabular}{|l|l|c|c|c|}
\hline \multicolumn{2}{|c|}{ Scale parameter } & 17 & 30 & 40 \\
\hline $\begin{array}{l}\text { Homogeneity } \\
\text { criterion }\end{array}$ & Shape factor & 0.2 & 0.2 & 0.3 \\
& Compactness & 0.4 & 0.5 & 0.5 \\
& No. of objects & 680 & 500 & 122 \\
\hline
\end{tabular}

Definiens Developer software mainly works on the concept of object-oriented classification but also works on the multiresolution segmentation. The image is segmented for the classification of different urban areas. Classification was done using a specific sets of rules. After being segmented, the image was queried with various parameters. Different sets of rules were applied on the segmented image using different parameters for extracting the buildings. So, the image was queried with various parameters for extracting the buildings. The best results of building extraction was achieved by using some spectral range, texture, values, and shape.

The segmentation was done at varying resolutions. This image segmentation technique is called multiresolution segmentation. This segmentation algorithm was applied on the image so that the similar kind of pixels form groups according to the applied homogeneity criteria. Thus, based on the homogeneity criteria, the objects were formed by merging the pixels falling under the criteria.

\section{Result of segmented images by different scale parameters}

By taking scale parameter 17 and homogeneity criteria (shape factor 0.2 , compactness $0.5)$, the objects are shown lying inside the building area in a segmented image. And this shows that these parameters are better for building extractions (Fig. 4). 
There are different parameters used for deciding the homogeneity criteria for the image segmentation. Multiresolution segmentation was done at different scale parameters. The scale parameters determine the size of the objects formed during segmentation.

By taking scale parameter 30 and shape factor 0.2 , compactness 0.5 , the objects of interest are shown lying outside the building-area boundary. It can be discerned that this parameter is not very suitable for building extraction (Fig. 5).
By taking scale parameter 40 , shape factor 0.3 , and compactness 0.5 , the objects are shown lying outside of the area of interest. This scale parameter is very helpful for extraction of big object like large building (Fig. 6).

Throughout the image segmentation, the whole image is segmented and image objects are generated based on several adjustable criteria of heterogeneity in shape. Modifying the value of the scale parameter varies the size of the resulting image objects. A highscale parameter results in large objects and
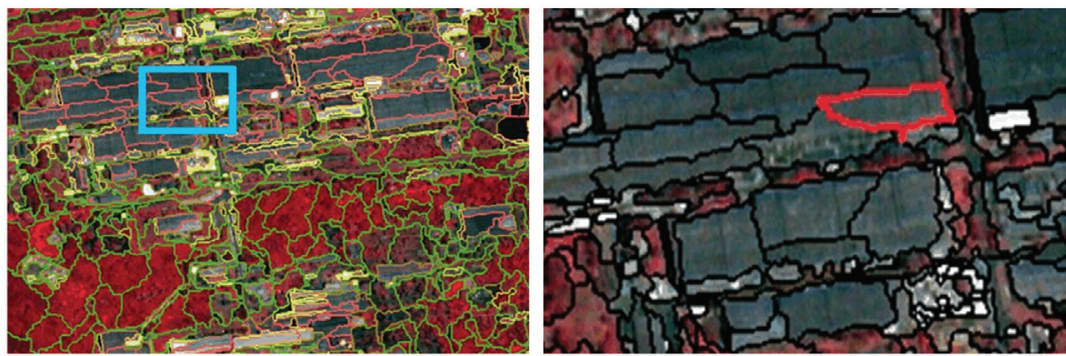

Figure 4. Result of a segmented image by scale parameter 17
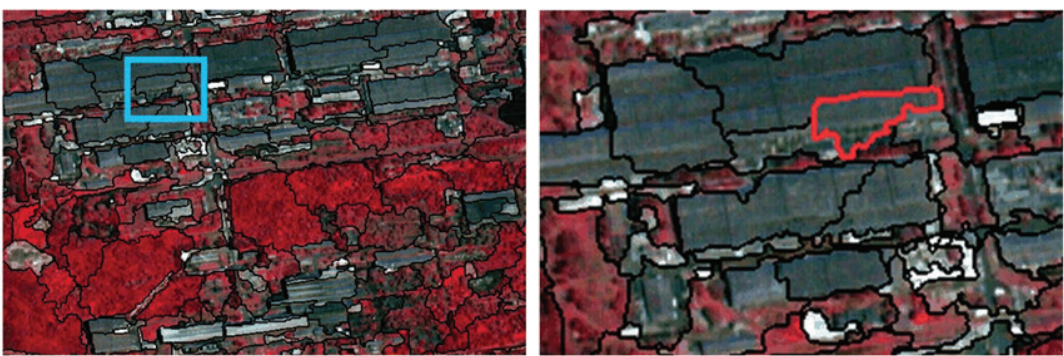

Figure 5. Result of the segmented image by scale parameter 30
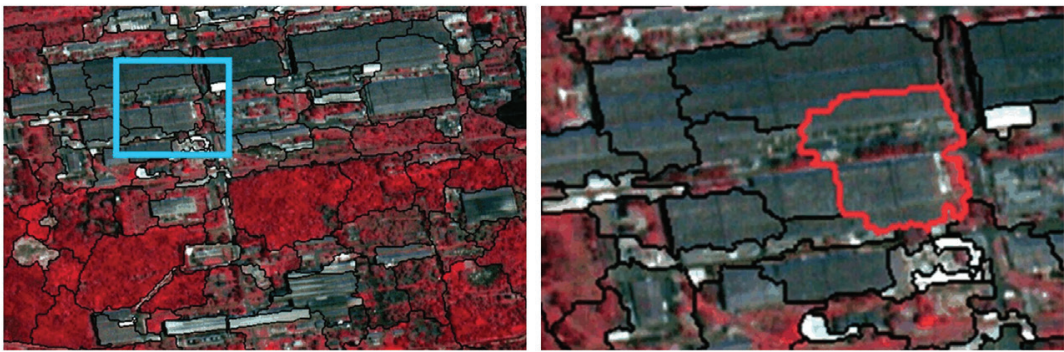

Figure 6 . Result of the segmented image by scale parameter 40 
vice-versa. After segmentation, the image is classified as an object-oriented approach. This process of classifying objects has been done using an export knowledge base which is an inbuilt function of Definiens Developer. Corresponding to the rule and the knowledge, the membership values and multiresolution fuzzy curves were defined. Different curves describe how the membership value for a specific expression is assigned and calculated for certain feature values of image objects. The fuzzy membership functions: Gaussian, Full range, Singleton, and Larger, proved the most promising for building extraction from the segmented image. A class description of vegetation, shadows, and buildings were analysed and the results are shown in Figures 7,8 , and 9 respectively.

\section{Class description of vegetation}

The segmentation-based classification is then applied to the image to call the desired classes to merge together to a new level. The cleaning process can be carried out in this new level and the classification of further details can be performed. The standard nearest neighbor classification can be carried out as well as the fuzzy logic function to assign those desired classes for exporting. The object-based classification results, which are only based on the spectral mean of the digital number itself, are not effective means to differentiate buildings with similar spectral values in the area.

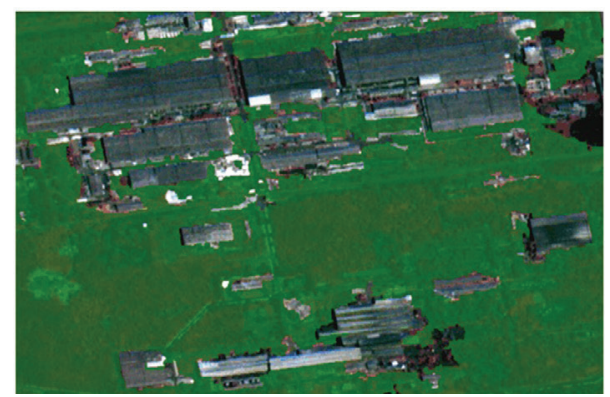

Figure 7. Classification of vegetated area and classification of vegetation class using algorithm classification

\section{Class description of shadow}

Different rules have been applied for building extraction. The class hierarchy generated for building extraction contains a set of object-based rules with their corresponding membership values. Every single rule was assigned to one membership function. These rules are collectively compiled in a class hierarchy and the image is classfied for building extraction.

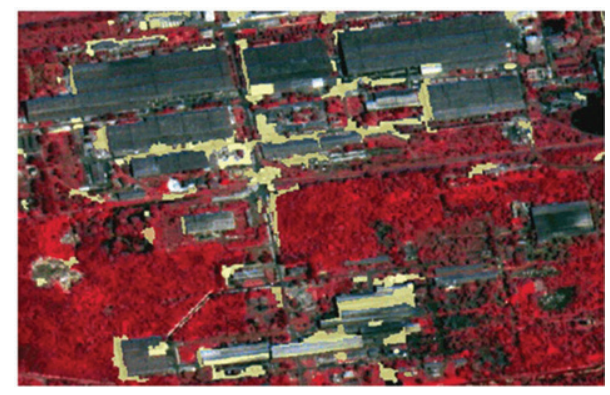

Figure 8. Classification of shadow and classification of shadow class, using algorithm classification

\section{Class description of buildings}

All classes and their contents are stored in the class hierarchy, and can be structured in a semantic way in the group hierarchy and inherit their conditions by structuring them in the inheritance hierarchy. The process tree window for the buildings, vegetation, shadows, and features of classification are also clearly represented, respectively.

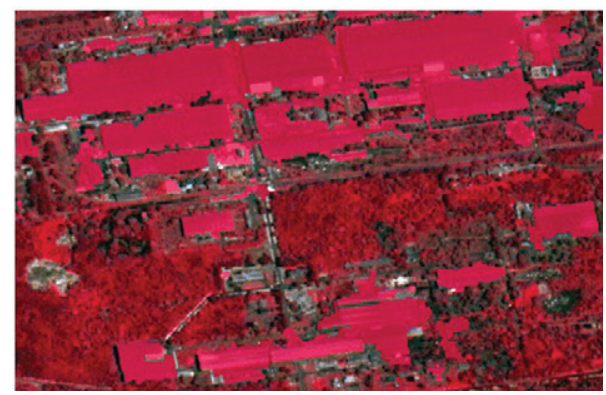

Figure 9. Classification of building and classification of building class, using algorithm classification 


\section{Accuracy measurement}

\section{Quantitative assessment}

The quantitative assessment was calculated based on area accuracy. It defines the accuracy of the extraction in the area, the difference in reference, and extracted data.

Area accuracy $=$ Area extracted (sq. m) / Area extracted (sq. m) $\times 100$

Table 4. Number of buildings and difference error

\begin{tabular}{|c|c|}
\hline No. of building & Difference error (\%) \\
\hline 1 & 1.09 \\
2 & 0.10 \\
3 & -3.66 \\
4 & 6.89 \\
5 & 14.30 \\
6 & -2.20 \\
7 & 7.71 \\
8 & 13.86 \\
9 & 12.70 \\
10 & 13.50 \\
11 & 7.08 \\
12 & -5.07 \\
13 & -3.13 \\
14 & -11.25 \\
\hline
\end{tabular}

Number of buildings and the percentage of difference error is clearly shown in Table 4. Building 5 shows maximum $14.30 \%$ difference error. Building 10 shows a $13.50 \%$ difference error. Buildings 3, 6, 12, 13, and 14 show a percentage difference error in a negative trend, in which building 14 shows a minimum $-11.25 \%$ difference error.

\section{Classified image}

After the classification process, a classified image was generated (Fig. 10). When we take the building from the classified image, we get only the feature of interest i.e. the buildings.

Overall accuracy of the classified image is 0.93 and Kappa accuracy is 0.89 . The produced accuracies for buildings, vegetation, and shadows were $0.9545,1.0$, and 0.8888 , respectively, whereas user accuracies for buildings, vegetation, and shadows were 1.0, 0.9375 , and 1.0 , respectively.

Overall classification accuracy based on the TTA mask (training and test area mask) was calculated. Overall classification accuracy was 0.97 and Kappa accuracy was 0.95. The producer-accuracy for buildings, vegetation, and shadows were 1.0, 1.0, and 0.7144, respectively, and user-accuracy for buildings, forests, and shadows were 1.0, 0.9375, and 1.0 , respectively.

The area of the referenced building was $14,728.46$ sq. $m$ whereas the area of the extracted buildings were $14,380.37$ sq. $\mathrm{m}$. The overall accuracy of the building is $97.80 \%$. Referenced buildings and extracted buildings are shown in Figure $11(a, b)$.

\section{Conclusion}

A building plays an important role in the urban scenario. An urban planner needs access
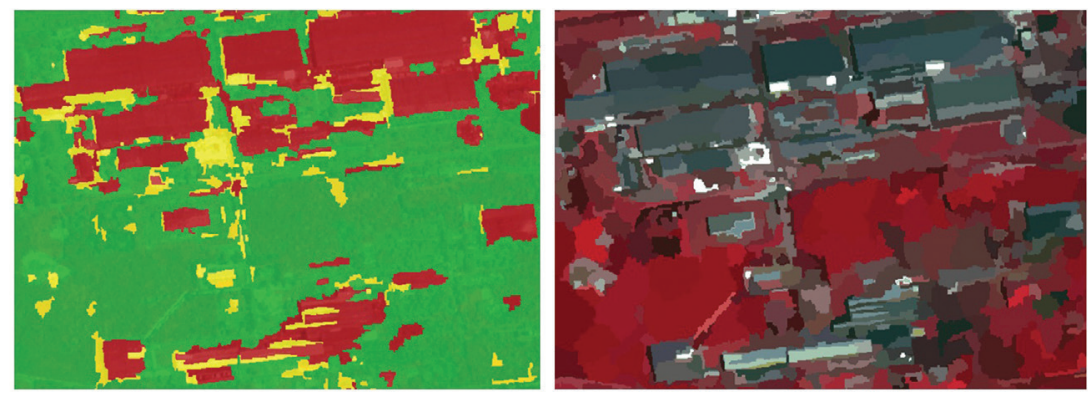

Figure 10. Steps for improving building extraction which generate a classified image after fuzzy classification and its accuracy 

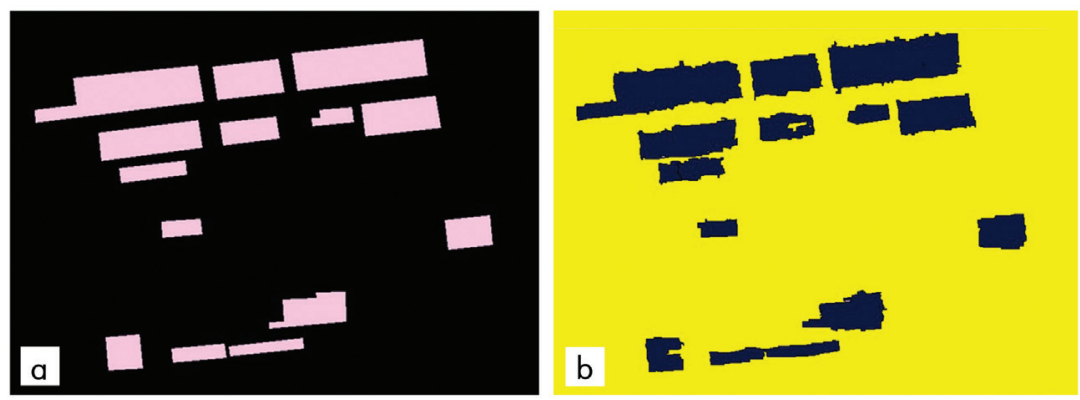

Figure 11. Referenced building and extracted buildings

to building data. High resolution satellite remote sensing data and related techniques can aid the urban planner. There are some problems with automatic building extraction using remotely sensed data This is especially true in urban areas due to the spectral complexity of the scene because buildings lying in a low and poor contrast image area could not be extracted using Cartosat-1 data. Some objects have a spectral reflectance which appears similar to buildings. Such an appearance is a hindrance when tone alone is used for extraction. Building roofs which are nonhomogeneous, sloping, flat etc. cause different spectral properties.

The steps followed in this study gave satisfactory result when compared with the original digitised vector layer. But, also, the clear edges of the buildings were not extracted; which coincides with the original image. So the clear edges can also be considered as urban buildings. The features extracted using the methods, which are not matching with the edges, also should be considered because they are at locations where they can be considered as the footprints of the buildings.

Image processing software like Erdas Imagine (ver. 10) helps to explore an image through image classification, and extracts the features by using a filtering process. The Definiens Developer has several options which also play an important role in the extraction of these features. When someone knows how to use this software, they can achieve suitable extractions according to the features. The fuzzy classification used in this software has many optional classifications to extract the features. The simple image processing software has its own limitations so that the feature extractions are not very easy. The edges of the building are extracted using the different high pass spatial filter but then the edges are not very sharp. So these images are to be thinned by using some thinning algorithms. Using automatic extraction techniques, there is an adavantage on the thinning of the edges of urban buildings extracted.

\section{Acknowledgement}

The first author heartily thanks Ms. Minakshi Kumar, Scientist-F, IIRS (ISRO), for offering her expertise, knowledge, and useful suggestions. The first author is also thankful to Ms. Shefali Agrawal, Scientist, IIRS (ISRO), Dehradiun, India, for her valuable suggestions.

Editors' note:

Unless otherwise stated, the sources of tables and figures are the authors' on the basis of their own research. 


\section{References}

Agouris P., Gyftakis S., Stefanidis A., 1998. Using a fuzzy supervisor for object extraction within an integrated geospatial environment. The International Archives of the Photogrammetry, Remote Sensing and Spatial Information Sciences, vol. 32, part 3, pp. 191-195.

BALTSAVIAS E.P., 1999. A comparison between photogrammetry and laser scanning. ISPRS Journal of Photogrammetry and Remote Sensing, vol. 54, no. 2-3, pp. 83-94.

Benediktsson J.A., Pesaresi M., Amason K., 2003. Classification and feature extraction for remote sensing images from urban areas based on morphological transformations. IEEE Transactions on Geoscience and Remote Sensing, vol. 41, no. 9, pp. 1940-1949.

BLASCHKE T., 2010. Object based image analysis for remote sensing. ISPRS Journal of Photogrammetry and Remote Sensing, vol. 65, no. 1, pp. 2-16.

Bruzzone L., Mingmin C., Marconcini M., 2006. A novel transductive SVM for Semi-supervised classification of remote-sensing images. IEEE Transactions on Geoscience and Remote Sensing, vol. 44, no. 11, pp. 3363-3373.

Burnett C., Blaschke T., 2002. Objects / notobjects and near-decomposability: ecosystems and GI [in:] M.J. Egenhofer, D.M. Mark (eds.), Geographic Information Science: Second international conference, GIScience 2002, Boulder, CO, USA, September 25-28, 2002: Proceedings, Berlin-New York: Springer, pp. 225-229.

BüCKNER J., 1998. Model based road extraction for the registration and interpretation of remote sensing data. The International Archives of the Photogrammetry, Remote Sensing and Spatial Information Sciences, vol. 32, part 4, pp. 85-90.

CHANG K.-T., 2007. Introduction to geographic information systems. New York: McGraw-Hill.

Chaudhuri D., Kushwaha N.K., Samal A., 2012. Semi-Automated road detection from high resolution satellite images by directional morphological enhancement and segmentation techniques. IEEE Journal of Selected Topics in Applied Earth Observations and Remote Sensing, vol. 5, no. 5, pp. 1538-1544.

CHEN T. (ed.), 1998. The past, present and future of image and multidimensional signal process- ing, IEEE Signal Processing Magazine, vol. 15, pp. 21-58.

Dell'Acqua F., Gamba P., 2007. Spectral Resolution in the Context of Very High Resolution Urban Remote Sensing [in:] Q. Weng, D. Quattrochi (eds.), Urban Remote Sensing. Boca Raton, FL: CRC/Taylor \& Francis, pp. 377-391.

Ding M.L., LI G.Y., Zhang Q.F., 2010. Application of road extraction in satellite images based on snakes model. Computer Technology and Development, vol. 1, no. 1, pp. 71-73.

Foresti G.L., Pellegrino F.A., 2004. Automatic visual recognition of deformable objects for grasping and manipulation. IEEE Transactions on Systems, Man, and Cybernetics. Part C: Applications and Reviews, vol. 34, no. 3, pp. 325-333.

Gonzalez R.C., Woods R.E., 2002. Digital image processing. Upper Saddle River: Prentice Hall.

GRUEN A., 2008. Reality-based generation of virtual environments for digital earth. International Journal of Digital Earth, vol. 1, no. 1, pp. 88-106.

Gupta N., Bhadauria H.S., 2014. Object based Information Extraction from High Resolution Satellite Imagery using eCognition. IJCSI International Journal of Computer Science Issues, vol. 11, no. 3/2, pp. 139-144.

Hay G.J., Castilla G., 2008. Geographic object-based image analysis (GEOBIA): A new name for a new discipline [in:] T. Blaschke, S. Lang, G.J. Hay (eds.), Object-Based Image Analysis, Berlin-Heidelberg: Springer, pp. 75-89.

Hu X.Y., Zhang Z.X., Zhang J.Q., 2002. Semiautomatic extraction of linear object from aerial image. Journal of Image and Graphics, vol. 7, no. 2, pp. 137-140.

Huertas A., Nevatia R., 1988. Detecting buildings in aerial images. Computer Vision, Graphics, and Image Processing, vol. 41, no. 2, pp. 131-152.

ItTI L., KoCH C., 2001. Computational modelling of visual attention. Nature Reviews. Neuroscience, vol. 2, no. 3, pp. 194-203.

JensEN J.R., 1996. Introductory Digital Image Processing: A Remote Sensing Perspective. Upper Saddle River: Prentice Hall.

JeOn B.K., Jang J.H., Hong K.S., 2002. Road detection in spaceborne SAR images using a genetic algorithm. IEEE Transactions on 
Geoscience and Remote Sensing, vol. 40, no. 1, pp. 22-29.

Kettig R.L., Landgrebe D.A., 1976. Classification of multispectral image data by extraction and classification of homogeneous objects. IEEE Transactions on Geoscience Electronics, vol. 14, no. 1, pp. 19-26.

KIM Z., Nevatia R., 1999. Uncertain reasoning and learning for feature grouping. Computer Vision and Image Understanding, vol. 76, no. 3, pp. 278-288.

Kwak E., Al-Durgham M., Habib A., 2012. Automatic $3 D$ building model generation from Lidar and image data using sequential minimum bounding rectangle. International Archives of the Photogrammetry, Remote Sensing and Spatial Information Sciences, vol. 39-B3, pp. 285-290.

Lee D.S., Shan J., Bethel J.S., 2003. Class-guided building extraction from Ikonos imagery. Photogrammetric Engineering \& Remote Sensing, vol. 69, no. 2, pp. 143-150.

Lhomme S., He D.-C., Morin D., 2004. Évaluation de la qualité d'une image Ikonos pour l'identification du bâti en milieu urbain. Télédétection, vol. 3, no. 5, pp. 457-466.

LiN C., Nevatia R., 1998. Building detection and description from a single intensity image. Computer Vision and Image Understanding, vol. 72, no. 2, pp. 101-121.

LuCCheSE L., Mitra S.K., 2001. Color image segmentation: A state-of-the-art survey. Proceedings of the Indian National Science Academy, vol. 67A, no. 2, pp. 207-221.

Matikainen L., Hyyppä J., Ahokas E., Markelin L., KaARTINEN H., 2009. An improved approach for automatic detection of changes in buildings. The International Archives of the Photogrammetry, Remote Sensing and Spatial Information Sciences, vol. 38, part 3-W8, pp. 61-67.

Mayer H., Laptev I., Baumgartner A., Steger C., 1997. Automatic road extraction based on Multi-Scale modeling, context, and snakes. The International Archives of the Photogrammetry, Remote Sensing and Spatial Information Sciences, vol. 32, part 3-2W3, pp. 106-113.

PAL N.R., PAL S.K., 1993. A review on image segmentation techniques. Pattern Recognition, vol. 26, no. 9, pp. 1277-1294.
Pandey A.K., 2004. Automatic urban road extraction using airborne laser scanning - altimetry and high resolution satellite data. Enschede: ITC (M.Sc. Thesis).

Pigeon L., Solaiman B., Toutin T., ThomSON K.P.B., 2001. Linear planimetric feature domains modeling for multisensors fusion in remote sensing. Proceedings of SPIE, Sensor Fusion: Architectures, Algorithms, and Applications IV, vol. 4051, pp. 8.

Puissant A., Weber C., 2002. The utility of very high spatial resolution images to identify urban objects. Geocarto International, vol. 17, no. 1, pp. 33-43.

Rizvi I.A., Mohan B.K., 2010. Object-oriented method for automatic extraction of road from High Resolution Satellite Images. Iranian Journal of Earth Sciences, vol. 2, no. 1, pp. 55-62.

RotTensteiner F., 2012. Advanced methods for automated object extraction from LiDAR in urban areas. IEEE International Geoscience and Remote Sensing Symposium. Proceedings, 22-27 July 2012, Munich, pp. 5402-5405.

Segl K., Kaufmann H., 2001. Detection of small objects from high-resolution panchromatic satellite imagery based on supervised image segmentation, IEEE Transactions on Geoscience and Remote Sensing, vol. 39, no. 9, pp. 20802083.

Shan J., LeE S.D., 2002. Generalization of building polygons extracted from IKONOS imagery. International Archives of the Photogrammetry, Remote Sensing and Spatial Information Sciences, vol. 34, part 4, pp. 297-304.

Tarsha-Kurdi F., Landes T., Grussenmeyer P., 2007. Hough-transform and extended Ransac algorithms for automatic detection of 3D building roof planes from Lidar data. International Archives of the Photogrammetry, Remote Sensing and Spatial Information Sciences, vol. 36, part 3-W52, pp. 407-412.

Teng X., Song S., Zhan Y., 2014. A Novel Road Extraction Algorithm for High Resolution Remote Sensing Images. Applied Mathematics \& Information Sciences, vol. 8, no. 3, pp. 1435$-1443$.

TSENG Y.-H., WANG S., 2003. Semiautomated building extraction based on CSG model-image fitting. Photogrammetric Engineering \& Remote Sensing, vol. 69, no. 2, pp. 171-180. 
Tupin F., Houshmand B., Datcu M., 2002. Road detection in dense urban areas using SAR imagery and the usefulness of multiple views. IEEE Transactions on Geoscience and Remote Sensing, vol. 40, no. 11, pp. 2405-2414.

UZAR M., 2014. Automatic building extraction with multi-sensor data using rule-based classification. European Journal of Remote Sensing, vol. 47, no. 8, pp. 1-18.

Zhang C., Murai S., Baltsavias E., 1999. Road network detection by mathematical morphology. Proceedings of ISPRS Workshop "3D Geospatial Data Production: Meeting Application Requirements", Zurich: Institute of Geodesy and Photogrammetry, pp. 185-200. 
http://rcin.org.pl 